\title{
IDENTIFICATION EN FRANCE D'UNE ENTÉROSEPTICÉMIE HÉMORRAGIQUE A YERSINIA RUCKERI (ENTERIC REDMOUTH DISEASE) CHEZ LA TRUITE ARC-EN-CIEL (SALMO GAIRDNERI RIDCHARDSON)
}

\author{
R. LeSEL (1), M. LeSEL (1), F. GAVINI (2), A. VUILLAUME (3)
}

\section{RÉSUMÉ}

Une maladie de la truite arc-en-ciel, connue sur le continent nord-américain et en Australie sous le nom de "Enteric red mouth disease" (ERM disease), a été mise en évidence pour la première fois en France. II s'agit d'une entérosepticémie hémorragique : l'agent pathogène est Yersinia ruckeri.

\section{ABSTRACT}

A disease of rainbow trout, known in North America and Australia as " Enteric red mouth disease", has been identified for the first time in France. It is an haemorrhagic enterosepticemia ; the causative pathogenic agent is Yersinia ruckeri.

Une maladie de la truite arc-en-ciel a été récemment identifiée dans le Sud-Ouest de la France. Cette maladie est connue sous le nom de "Enteric red mouth disease "; elle a été décrite pour la première fois par RUCKER (1966) dans la vallée d'Hagerman (IDAHO. USA). Endémique en Amérique du Nord (BUSCH, 1978), elle existe aussi en Australie (BULLOCK et al., 1978) mais n'a, jusqu'à présent, pas été objectivement reconnue en France.

Une description complète des caractères de la maladie a été donnée par BUSCH (1978, 1982). Les observations que nous rapportons ont été réalisées dans une pisciculture du Sud-Ouest de la France. Les premiers signes caractéristiques de l'affectation sont apparus en mars 1981 chez des sujets de $8 \mathbf{g}$; une semaine plus tard, une bande de truitelles de $55 \mathrm{~g}$ présentaient une symptomatologie identique. Très vite, l'ensemble de l'exploitation était contaminé et toutes les classes d'âge présentes sur la pisciculture présentaient les mêmes signes cliniques. La maladie avait un caractère apparemment cyclique pendant la période chaude de l'année, des périodes de rémissions de 5 à 7 semaines étant ponctuées de flambées épidémiques. La mortalité hebdomadaire lors de ces flambées est de $1 \%$ des sujets contre moins de $0,1 \%$ entre les phases épidémiques.

(1) Institut National de la Recherche Agronomique, Laboratoire des Microorganismes, Centre de Recherches Hydrobiologiques - BP 3 Saint-Pée sur Nivelle 64310 ASCAIN.

(2) Institut National de la Santé et de la Recherche Médicale, Unité n० 146 , Ecotoxicologie microbienne - CERTIA BP 3959651 VILLENEUVE D'ASCO.

(3) Direction Départementale des Services Vétérinaires, rue Pasteur, 40000 MONT DE MARSAN. 
Au cours de celles-ci, le comportement des sujets est modifié : nervosité suivie de troubles de l'équilibre et de léthargie. Les sujets malades sont mélaniques et présentent souvent une exophtalmie unilatérale ou bilatérale qui peut évoluer jusqu'à la perte complète du globe oculaire. Les manifestations cliniques sont essentiellement hémorragiques : hémorragies sous-cutanées, à la base des nageoires, autour des yeux et de l'anus, sur les branchies, dans la bouche, sous la langue. A l'autopsie, on observe des pétéchies dans la graisse, sur le foie, le pancréas, les appendices pyloriques, l'intestin et la vessie natatoire: l'estomac est œdémateux.

L'agent responsable a été isolé et identifié comme étant Yersinia ruckeri, espèce décrite par EWING et al. (1978). Son identification sur plaque API 20 E suggère des identités variables : Hafnia alvei, Serratia sp. Une étude spécifique de 6 souches isolées au cours de différentes épidémies en 1981 et 1982 a été réalisée. Ces souches ont été soumises à un système informatisé d'identification des entérobactéries à $\beta$ galactosidase positive, système utilisant 24 caractères biochimiques (LEFEBVRE et GAVINI, 1982; GAVINI et al., 1982). Les 6 souches ont été identifiées comme étant Yersinia ruckeri.

Cette identification, associée à une symptomatologie conforme aux descriptions de BUSCH (1978, 1982) et RUCKER (1966). établit le diagnostic d'Entérosepticémie hémorragique (ERM disease). II s'agit de la première identification de cette maladie en France.

Compte tenu de cette mise en évidence et des confusions possibles de la bactérie responsable avec d'autres espèces bactériennes (en particulier Hafnia a/vei), nous avons revu les résultats d'analyses effectuées à l'occasion de bactérioses dans diverses piscicultures du Sud-Ouest de la France. Les symptômes cliniques et les lésions macroscopiques étaient souvent associés à un germe pathogène possédant les caractéristiques biochimiques de Yersinia ruckeri. II est donc probable que cette entérosepticémie hémorragique est plus fréquente qu'il ne le paraît.

\section{REFÉRENCES BIBLIOGRAPHIQUES}

BULLOCK G.L., STUKEY H.M., SHOTTS E.B. Jr., 1978. Enteric redmouth bacterium : comparison of isolates from different geographic areas. J. Fish Dis., 1, 351-354.

BUSCH R.A., 1978. Enteric Red Mouth disease (Hagerman strain). Mar. Fish. Rev., 40, 42-51.

BUSCH R.A., 1982. Enteric Redmouth disease (Yersinia ruckeri). In : Antigens of Fish Pathogens. Development and Production of Vaccines and Serodiagnostics. Symp. Int., Fondation Mérieux, Talloires (France), 22 p.

EWING W.H., ROSS A.J., BRENNER D.J., FANNING G.R., 1978. Yersinia ruckeri sp. nov. the Redmouth (DM) Bacterium. Int. J. Syst. Bacteriol., 28, 37-44.

GAVINI F., OGER C., LEFEBVRE B., IZARD D., LECLERC H., 1982. Development of a computer identification system for coliform strains. J. Appl. Bacteriol., 52, 329-332.

LEFEBVRE B., GAVINI F., 1982. Theory and programming of a computer identification system for coliform strains. J. Appl. Bacteriol., 52, 325-328.

RUCKER R.R., 1966. Redmouth disease of rainbow trout (Salmo gairdneri).Bull. Off. int. Epizoot., 65, 825-830. 